\title{
Editorial
}

\section{Trigeminocardiac reflexes}

Victor Faria Blanc MD FRCPC

Trigeminocardiac and trigeminorespiratory relexes were first described by Kratschmer (1870) in the cat and the rabbit. ' Angell-James and Daly (1969), ${ }^{2-3}$ confirmed that in dogs, under pentobarbital or chloralose-urethane anaesthesia, stimulation of the nasal mucous membrane (with cold water, tobacco smoke, or an electrical square wave stimulus of 2-5 volts, 1 msec duration, and 10-20 $\mathrm{Hz}$ ) caused bradypnoea and/or expiratory apnoea with bradycardia and variable changes in blood pressure. All these responses were abolished by the application of local anaesthetic to the nose, or by division of the trigeminal nerves which form the afferent pathway for such reflexes.

Modern reconstructive plastic surgery, permitting surgical correction of craniofacial and maxillofacial deformities, was initiated by Tessier et al. in $1967 . .^{4-5}$ These new surgical procedures created new anaesthetic problems and surgical complications. Since 1978, 15 cases of sudden bradycardia and/or asystole during cranio-maxillo-facial surgery have been reported. ${ }^{6-15}$ In this issue, Lang $e t$ $a l .{ }^{16}$ present three additional cases confirming that reflex bradycardic responses can occur after stimulation of sensory branches of the maxillary $\left(\mathrm{V}_{2}\right)$ and mandibular $\left(V_{3}\right)$ divisions of the trigeminal nerve. In 1987, Bainton and Lizi, ${ }^{7}$ and Loewinger et al. ${ }^{8}$ suggested that trigeminocardiac reflexes (TCR) can be elicited by stimulating afferent paths other than the ciliary nerves (branches of the ophthalmic division $-V_{1}-$ of the trigeminal nerve) which are classically associated with the oculocardiac reflex (OCR).

\section{Evidence for causality}

The criteria necessary to establish a cause-and-effect relationship include the consistency and the strength of the association, the presence of a dose-response (or type of stimulus-incidence) relationship, and biological plausibility. ${ }^{17-18}$

In each one of the eighteen cases reported, ${ }^{6-16}$ the cause (surgical manipulation of bony cranio-maxillo-

\footnotetext{
Département d'Anesthésie-réanimation de l'Université de Montréal, Hópital Sainte-Justine, Montréal, Québec.

Accepted for publication 4ih April, 1991.
}

facial structures or of the attached soft tissues) elicited a very specific and unequivocal effect (sudden bradycardia, junctional rhythm, or sinus arrest). Each time the cause was eliminated the effect resolved and, in five of the cases, $9,13,16$ repeating the cause elicited the same effect.

The cause-effect association can be evaluated further by examining the effectiveness of the measures used to prevent the response. The reflex can be prevented by avoiding stimulation of the afferent path. This can be achieved by modulating the surgical stimulus ${ }^{20}$ and/or by blocking the sensory nerves that conduct the afferent impulses. In this issue, Lang et al. ${ }^{16}$ consider neural blockade in the prevention of TCR. However, most recommend the use of intravenous anticholinergics to block the muscarinic receptors of the heart and to prevent the occurrence of bradycardia. ${ }^{7-9,13-15}$ In nine of the reported cases, ${ }^{7,9,13-16}$ atropine, $0.6 \mathrm{mg}$ iv, or glycopyrrolate, $0.2-0.5 \mathrm{mg} i v$, was given to prevent the recurrence of the cardiac dysrhythmia. In each case, anticholinergic medication was an effective prophylactic measure because no further episodes of dysrhythmia were encountered and the surgical procedures could be completed uneventfully.

The type of stimulus may modify the TCR response. For example, as electrical stimulation of the vagus nerve is increased up to frequencies of $20-30 \mathrm{~Hz}$, the SA node becomes progressively more inhibited. But at higher frequencies the vagal response may decrease. When the stimulation stops, the heart rate rapidly retums to the control level, reflecting the rapid hydrolysis of the acetylcholine that had been liberated during stimulation. ${ }^{19}$ As far as the OCR is concerned, it has been shown that abrupt and sustained traction (type A stimuli) is more reflexogenic than smooth and gentle traction (type B stimuli). ${ }^{20}$ It is expected that changing the stimulus modifies the TCR relationship although there is no mention of the type of stimulus in clinical reports dealing with TCR other than the OCR.

Laboratory investigations in the rabbit, the cat, and the dog, ${ }^{1-3}$ clinical experience with the OCR, ${ }^{20,23}$ clinical reports of TCR other than OCR, ${ }^{6-16}$ and systematic application of knowledge of the anatomical distribution of the trigeminal nerve, ${ }^{7,11,21,22}$ support the assumption that 
TABLE Afferent pathways other than the ciliary nerves (OCR) involved in TCR

\begin{tabular}{lll}
\hline Sensory nerves andior branches & Trigeminal division & References \\
\hline Frontal and supratrochlear nerves & $V_{1}$ & 12 \\
Recurrent tentorial branches & $V_{1}$ & 11 \\
Maxillary nerve branches & $V_{2}$ & $10,13-16$ \\
Zygomaticotemporal branches & $V_{2}$ & $7-9,14^{*}$ \\
Superior alveolar branches & $V_{2}$ & 12 \\
Auriculotemporal nerve & $V_{3}$ & $7-9,14^{*}$ \\
Inferior alveolar nerve & $V_{3}$ & 16 \\
\hline
\end{tabular}

$V_{1}=$ ophthalmic division; $V_{2}=$ maxillary division; $V_{3}=$ mandibular division.

*Note that the sensory supply to the zygomatic arch area is through the zygomaticotemporal branches of $\mathrm{V}_{2}$ anteriorly and through the temporal branches of the auriculotemporal nerve $\left(V_{3}\right)$ posteriorly, $7,21-22$

stimulation of any sensory branch of the $V$ th cranial nerve may lead to severe bradycardia and/or ventricular asystole.

Afferent paths other than the ciliary nerves (OCR) most frequently involved in TCR

Unfortunately, several case reports are too imprecise to allow identification of the sensory nerve involved in the afferent conduction of the TCR. Nevertheless, it is possible to construct the Table.

\section{Prophylaxis and treatment of TCR}

The risk of TCR should be considered in any craniomaxillo-facial surgical intervention. A knowledge of the applied anatomy helps in predicting this risk and in choosing appropriate prophylaxis. If controlled hypotension is planned, the prophylaxis of TCR is better accomplished with local anaesthetic infiltration or block of the nerve(s) which convey afferent stimuli. If there is no contra-indication to intravenous anticholinergics, atropine and/or glycopyrrolate IV may be used to prevent a TCR.

The treatment of TCR deserves more attention. If a TCR is elicited, the surgeon must stop the stimulus and wait until the pulse recovers its normal rhythm. It is dangerous to use an anticholinergic iv before the stimulus has been stopped and the cardiac dysrhythmia has resolved because of the risk of converting sinus bradycardia into premature ventricular contractions and runs of bigeminy. $7,9,20,23$ Once the heart rate has returned to normal, neural blockade and/or intravenous anticholinergics are indicated to prevent recurrence of TCR. Finally, the surgeon should continue the operation with gentler, less reflexogenic, manipulation.

Controlled ventilation is recommended for the prophylaxis and treatment of TCR. Hypercapnia augments the incidence of the OCR in spontaneously breathing infants and children ${ }^{20}$ and hypoxaemia must be prevented when facing the possibility of cardiac arrhythmias. These ventilatory abnormalities may be due to, or aggravated by, a trigeminorespiratory reflex (TRR). Trigeminorespiratory reflexes are commonly associated with TCR. They can be elicited in animals ${ }^{1-3}$ and in man. ${ }^{24}$ Also, the diving reflex in seals and other diving vertebrates results from trigeminal afferent activity. ${ }^{25-26}$

During cranio-maxillo-facial surgery TCR should be anticipated and prophylactic steps should be taken.

\section{Les réflexes trigéminocardiaques}

Les premières descriptions de réflexes trigéminocardiaques et trigéminorespiratoires, provoqués chez le chat et le lapin, sont celles de Kratschmer (1870). ' AngellJames et Daly $(1969)^{2-3}$ ont confirmé que, chez des chiens anesthésiés à l'aide de pentobarbital ou de chloralose-uréthane, la stimulation de la muqueuse nasale (avec de l'eau froide, de la fumée de tabac, ou des stimuli électriques d'onde carrée, de 2-5 volts, durant 1 msec, et à des fréquences de $10-20 \mathrm{~Hz}$ ) déclenchait une bradypnée et/ou une apnée en expiration, avec bradycardie et des variations inconstantes des pressions artérielles. Toutes ces réponses étaient abolies par l'anesthésie de la muqueuse nasale ou par la section des nerfs trijumeaux qui constituent, donc, la voie afférente de ces réflexes.

La chirurgie plastique et reconstructive moderne, permettant de corriger des malformations craniofaciales et maxillofaciales, a été initiée par Tessier et al. ${ }^{4-5}$ en 1967. Ces nouvelles procédures chirurgicales ont été à l'origine de nouveaux problèmes anesthésiques et de nouvelles complications peropératoires. Depuis 1978, quinze cas de bradycardie subite et/ou d'asystolie ventriculaire, survenant au cours d'interventions de chirurgie cranio-maxillo-faciale, ont été rapportés. ${ }^{6-15}$ Dans ce numéro, Lang el al. ${ }^{16}$ présentent trois autres cas cliniques qui confirment que des bradycardies d'origine réflexe peuvent être déclenchées par la stimulation de branches sensitives du nerf maxillaire supérieur $\left(V_{2}\right)$, ou du nerf maxillaire inférieur $\left(V_{3}\right)$, du trijumeau. En 1987, Bainton et Lizi, ${ }^{7}$ de même que Loewinger et al. ${ }^{8}$ ont suggéré que des réflexes trigéminocardiaques (RTC) peuvent être déclenchés par la stimulation chirurgicale de voies afférentes autres que les nerfs ciliaires (branches du nerf 
ophtalmique $-V_{1}-$ du trijumeau) classiquement reliés au réflexe oculocardiaque (ROC).

\section{Évidences en faveur de la causalité}

Les critères qui nous permettent d'évaluer une association cause-effet comprennent la consistance et la solidité de cette-même association, la présence d'une relation dose-effet (ou type de stimulus - incidence) et la plausibilité biologique. ${ }^{17-18}$

Chez chacun des dix-huit cas cliniques qui ont été rapportés, ${ }^{6-16}$ la cause (manipulation chirurgicale de structures osseuses cranio-maxillo-faciales ou des tissus mous qui s'y attachent) a déclenché un effet et spécifique et non-équivoque (bradycardie subite, ou rythme jonctionnel, ou arrêt sinusal). Chaque fois que la cause était arrêtée l'effet se dissipait en quelques secondes. Chez cing de ces cas cliniques, ${ }^{9,13,16}$ la répétition de la cause produisait la répétition du même effet.

Nous pouvons approfondier notre analyse de l'association cause-effet en examinant l'efficacité des mesures prophylactiques qui ont été utilisées lorsqu'il y a eu un RTC positif. Le réflexe peut être évité en évitant de stimuler sa voie afférente. Cet objectif peut être atteint en modulant le stimulus chirurgical ${ }^{20}$ et/ou en bloquant les nerfs sensitifs qui conduisent l'influx afférent. Dans ce numéro, Lang et al. ${ }^{16}$ considèrent l'intérêt prophylactique du blocage des nerfs sensitifs. Cependant, la majorité des auteurs a préféré le recours aux anticholinergiques, par voie intraveineuse, dans le but de bloquer les récepteurs muscariniques du cœur et de prévenir ainsi l'occurrence d'épisodes de bradycardie. ${ }^{7-9,13-15}$ Chez neuf des cas rapportés, ${ }^{7,9,13-16}$ l'atropine, $0,6 \mathrm{mg}$ iv, ou le glycopyrrolate, $0,2-0,5 \mathrm{mg} i v$, ont été utilisés dans le but d'éviter la récidive de dysrythmies cardiaques. Dans tous ces cas, la médication anticholinergique s'est avérée efficace dans la mesure où elle a éliminé la récidive de dysrythmies cardiaques et a permis de terminer les interventions chirurgicales sans incidents.

Le type de stimulus peut modifier l'incidence de RTC. Par exemple, en augmentant la fréquence de la stimulation vagale on augmente, progressivement, son effet d'inhibition au niveau du nœud SA. Jusqu'à des fréquences de $20-30 \mathrm{~Hz}$, la fréquence cardiaque diminue au fur et à mesure que la stimulation vagale augmente. À des fréquences plus élevées, la réponse vagale peut diminuer. Assitôt que l'on cesse cette stimulation la fréquence cardiaque revient rapidement à la normale, reflétant ainsi la rapidité de l'hydrolyse de l'acétylcholine qui a été libérée pendant la phase de stimulation. ${ }^{19}$ Pour ce qui est du ROC, il a été démontré que les tractions brusques et soutenues (stimuli du type A) sont plus réflexogènes que les tractions progressives et délicates (stimuli du type B) ${ }^{20}$ Ceci dit, il faudra s'attendre à ce que le type de stimulus conditionne l'incidence de RTC positifs.
TABLEAU Voies afférentes autres que les nerfs ciliaires (ROC) impliquées dans des RTC

\begin{tabular}{lll}
\hline Nerfs ellou branches sensitives & Branches du trijumeau & Réferences \\
\hline $\begin{array}{l}\text { Nerfs frontal et supratrochléaire } \\
\text { Branches pour la tente du } \\
\text { cervelet }\end{array}$ & $v_{1}$ & 12 \\
$\begin{array}{l}\text { Branches du nerf maxillaire } \\
\text { supérieur }\end{array}$ & $v_{1}$ & 11 \\
$\begin{array}{l}\text { Branches du nerf zygomato- } \\
\text { temporal }\end{array}$ & $V_{2}$ & $10,13-16$ \\
$\begin{array}{l}\text { Nerf dentaire supérieur } \\
\text { Nerf auriculo-temporal }\end{array}$ & $V_{2}$ & $7-9,14^{*}$ \\
Nerf dentaire inférieur & $V_{2}$ & 12 \\
\hline
\end{tabular}

$V_{1}=$ nerf ophalmique; $V_{2}=$ nerf maxillaire supérieur; $V_{3}=$ nerf maxillaire inférieur.

*Notez que l'innervation sensilive de l'arcade zygomatique comprend des branches du nerf zygomato-temporal $\left(V_{2}\right)$, antérieures, et des branches temporales du nerf auriculo-temporal $\left(V_{3}\right)$, postérieures. ${ }^{7,21-22}$

Les travaux de recherche de laboratoire chez des lapins, des chats, et des chiens, ${ }^{1-3}$ l'experrience clinique accumulée autour du $\mathrm{ROC}^{20,23}$ les rapports de cas cliniques dédiés à des RTC autres que le ROC, ${ }^{6-16}$ et l'anatomie topographique et fonctionnelle du nerf trijumeau, $, 11,21-22$ nous permettent d'admettre que la stimulation de n'importe quelle branche sensitive du trijumeau peut induire une bradycardie sévère evou une asystolie ventriculaire.

Voies afférentes autres que les nerfs ciliaires (ROC) le plus souvent impliquées dans des RTC

Malheureusement, plusieurs rapport cliniques sont beaucoup trop imprécis pour que l'on puisse identifier la ou les branches sensitives du trijumeau éventuellement impliquées dans des RTC. Néanmoins, il est possible de construire le Tableau suivant.

\section{La prophylaxie et le traitement des RTC}

Un bon protocole de chirurgie cranio-maxillo-faciale doit inclure l'évaluation du risque de RTC. L'anatomie topographique et fonctionnelle aide à prédire ce risque et à choisir des blocages nerveux prophylactiques. Si l'hypotension contrôlée est indiquée, il sera préférable de recourir à l'anesthésie locale ou loco-régionale, dans le but d'éviter un RTC. S'il n'y a pas de contre-indication à l'usage des anticholinergiques, l'atropine et/ou le glycopyrrolate iv peuvent être choisis pour la prophylaxie des RTC.

Le traitement des RTC éxige un peu plus d'attention. Si un RTC a été déclenché, le chirurgien doit arrêter la stimulation chirurgicale, et attendre, jusqu'à ce que le pouls récupère un rythme normal. Il est dangereux d'administrer un anticholinergique iv avant que le stimulus chirurgical ne soit arrêté et que la dysrythmie 
cardiaque ne soit disparue. Autrement, il est fort probable qu'une bradycardie sinusale se convertisse en extrasystoles ventriculaires et/ou en rythme bigéminé. ${ }^{7,9,20,23}$ Lorsque le rythme cardiaque retourne à la normale, les blocages nerveux et/ou l'administration d'un anticholinergique iv redeviennent indiqués pour éviter la récidive d'un RTC. Finalement, il est important que le chirurgien poursuive l'intervention en employant des gestes beaucoup plus délicats, c'est-à-dire, beaucoup moins réflexogènes.

La ventilation contrôlée est à recommander et pour la prophylaxie et pour le traitement des RTC. L'hypercapnie augmente l'incidence de ROC positifs chez les nourrissons et chez les enfants anesthésiés sous respiration spontanée ${ }^{20}$ et l'hypoxémie doit être évitée lorsque l'on envisage la possibilité d'arythmie cardiaque. Ces anomalies ventilatoires peuvent être dues à, ou aggravées par un réflexe trigéminorespiratoire (RTR). Les réflexes trigéminorespiratoires sont fréquemment associés aux RTC. Ils peuvent être facilement déclenchés et chez les animaux de laboratoire ${ }^{1-3}$ et chez les êtres humains. ${ }^{24}$ En plus, chez les foques et chez d'autres vertébrés plongeurs, l'immersion de la tête déclenche une apnée et une bradycardie reflexes qui sont dues à la stimulation de branches sensitives due trijumeau. ${ }^{25-26}$

Pendant la chirurgie cranio-maxillo-faciale, il faut être attentif et tout à fait prêt à prévoir, à éviter et à traiter un RTC.

\section{References/Références}

1 Kratschmer $F$. Über Reflexe von der Nasenschleimhaut auf Athmung und Kreislauf. Sber Akad Wis Wien 1870; 62: 147-70.

2 Angell-James JE, Daly MB. Nasal reflexes. Proc Roy Soc Med 1969; 62: 1287-93.

3 Angell-James JE, Daly $M B$. Some aspects of upper respiratory tract reflexes. Acta Otolaryngol 1975; 79: 242-52.

4 Tessier P, Guiot G, Rougerie J, Delbet JP, Pastoriza $J$. Ostéotomies cranio-naso-orbito-faciales. Hypertélorisme. Ann Chir Plast 1967; 12: 103-18.

5 Tessier $P$. Ostéotomies totales de la face. Syndrome de Crouzon. Syndrome d'A pert. Oxycéphalies. Scaphocéphalies. Turricéphalies. Ann Chir Plast 1967; 12: 273-86.

6 Robideaux $V$. Oculocardiac reflex caused by midface disimpaction. Anesthesiology 1978; 49: 433.

7 Bainton R, Lizi E. Cardiac asystole complicating zygomatic arch fracture. Oral Surg Oral Med Oral Pathol 1987; 64: 24-5.

8 Loewinger $J$, Cohen $M$, Levi $E$. Bradycardia during elevation of a zygomatic arch fracture. J Oral Maxillofac Surg 1987; 45: 710-1.

9 Shearer ES, Wenstone R. Bradycardia during elevation of zygomatic fractures - a variation of the oculocardiac reflex. Anaesthesia 1987; 42: 1207-8.

10 Shelly MP, Church JJ. Bradycardia and facial surgery (letter). Anaesthesia 1988; 43: 422.

11 Hopkins CS. Bradycardia during neurosurgery - a new reflex? (Letter). Anaesthesia 1988; 43: 157-8.

12 Stott $D G$. Reflex bradycardia in facial surgery. Br J Plast Surg 1989; 42: 595-7.

13 Ragno JR, Marcoot RM, Taylor SE. Asystole during Le Fort I osteotomy. J Oral Maxillofac Surg 1989; 47: 1082-3.

14 Barnard NA, Bainton R. Bradycardia and the trigeminal nerve. J Cranio-Max-Fac Surg 1990; 18: 359-60.

15 Bainton R, Barnard NA, Wiles JR, Bice J. Sinus arrest complicating a bitemporal approach to the treatment of pan-facial fractures. Br J Oral Maxillofac Surg 1990; 28: 109-10.

16 Lang $S$, Lanigan $D T$, van der Wal $M$. Trigeminocardiac reflexes: maxillary and mandibular variants of the oculocardiac reflex. Can J Anaesth 1991; 38: 757-60.

17 Huley $S B$, Cummings SR. Designing Clinical Research, Baltimore: Williams \& Wilkins 1988.

18 Jenicek $M$, Cléroux $R$. Épidémiologie: Principes, Techniques, Applications, St-Hyacinthe, Quebec: Edisem 1987.

19 Levy M, Martin PL. Neural control of the heart. In: Bern RM, Sperelakis N, Geiger SR (Eds.). Handbook of Physiology, The Cardiovascular System, Vol I, The Heart, Bethesda: American Physiological Society 1979: $581-620$.

20 Blanc VF, Hardy J-F, Milot J, Jacob J-L. The oculocardiac reflex: a graphic and statistical analysis in infants and children. Can Anaesth Soc J 1983; 30: 360-9.

21 Clemente $C D$. Gray's Anatomy of the Human Body, 30th American edit., Philadelphia: Lea \& Febiger 1985.

22 Rouvière H, Delmas A. Anatomie Humaine: Descriptive, Topographique et Fonctionnelle, Tome 1, Tête et Cou, I lème édit. Paris: Masson et Cie Éditeurs 1974.

23 Schwartz $H$. Oculocardiac reflex: is prophylaxis necessary? In: Mark LC, Ngai SH (Eds.). Highlights of Clinical Anesthesiology, New York: Harper and Row 1971: 111-4.

24 Blanc VF, Jacob J-L, Milor J, Cyrenne L. The oculorespiratory reflex revisited. Can J Anaesth 1988; 35: 468-72.

25 Tenney SM, Boggs DF. Comparative mammalian respiratory control. In: Fishman AP ef al. (Eds.). Handbook of Physiology, The Respiratory System, Vol II, Control of Breathing, Part 2, Bethesda: American Physiological Society 1986: 833-55.

26 Anderson HT. Physiological adaptations in diving vertebrates. Physiol Rev 1966; 46: 212-43. 